J. DYSZLEWICZ (Wrocław)

\title{
STRESS EQUATIONS OF MOTION OF IGNACZAK TYPE FOR THE SECOND AXISYMMETRIC PROBLEM OF MICROPOLAR ELASTODYNAMICS
}

Abstract. A second axially-symmetric initial-boundary value problem of linear homogeneous isotropic micropolar elastodynamics in which the displacement and rotation take the forms

$$
\underline{u}=\left(0, u_{\theta}, 0\right), \quad \underline{\varphi}=\left(\varphi_{r}, 0, \varphi_{z}\right)
$$

$((r, \theta, z)$ are cylindrical coordinates; $\mathrm{cf}$. [17]) is formulated in a pure stress language similar to that of [12]. In particular, it is shown how $\underline{u}$ and $\underline{\varphi}$ can be recovered from a solution of the associated pure stress initial-boundary value problem, and how a singular solution corresponding to harmonic vibrations of a concentrated body couple in an infinite space can be obtained from the solution of a pure stress problem.

1. Introduction. It was shown in [7, p. 212] (cf. also [16, p. 511] and [11]) that a mixed initial-boundary value problem of classical linear elastodynamics can be characterized in terms of stresses only. Such a characterization was extended to micropolar elastodynamics in cartesian coordinates by Ignaczak [12]. Closely related to the subject are the pure stress field equations presented by Iesan [9] and Olesiak [18].

In the present paper we describe a pure stress initial-boundary value problem of micropolar elastodynamics in cylindrical coordinates that corresponds to the second axially-symmetric problem of the theory. Also, we use the pure stress formulation to present a singular solution of micropolar elastodynamics for an infinite space.

1991 Mathematics Subject Classification: Primary 73D20.

Key words and phrases: micropolar elasticity theory, stress equations of motion problem (SEMP). 
2. Basic equations of micropolar elastodynamics for the second axisymmetric problem. A general linear theory of Cosserat continuum was proposed, among others, in [1], [6] and [17]. In the present paper a particular continuum of Cosserat type, a so-called micropolar elastic body, is discussed (cf. [16, p. 709]). For such a body an elastic process may be described by two independent vector fields: the displacement vector $\underline{u}$ and the rotation vector $\varphi$ (cf. [17, p. 10$]$ ). In a cylindrical system of coordinates $(r, \theta, z)$, and for the second axially-symmetric problem, these two vector fields depend on the space variables $r$ and $z$, and on time $t$ only, and they take the particular form

$$
\underline{u}=\left(0, u_{\theta}, 0\right), \quad \underline{\varphi}=\left(\varphi_{r}, 0, \varphi_{z}\right) \quad \text { in } \Omega \times(0, \infty),
$$

where $\varphi_{r}, u_{\theta}$ and $\varphi_{z}$ depend on $(r, z, t)$ only. In (2.1), $\Omega$ is a domain in $\mathbb{E}^{3}$ occupied by the body and $(0, \infty)$ is the time interval. It is assumed that the body is homogeneous and isotropic. Therefore, it can be identified with a function $\Omega=\Omega(\mu, \alpha, \beta, \gamma, \varepsilon, \varrho, J)$, where $\mu, \alpha, \beta, \gamma, \varepsilon, \varrho$ and $J$ are material constants. In the following $\bar{\Omega}=\Omega \cup \partial \Omega$, where $\partial \Omega$ is a smooth boundary of $\Omega$. Also, for a differentiable function $f=f(r, z, t)$ we introduce the notations: $\partial_{r} f=\partial f / \partial r, \partial_{z} f=\partial f / \partial z$, and $\dot{f}=\partial f / \partial t$. We write $f \in C^{n, m}[\Omega \times(0, \infty)]$ whenever $f(\cdot, \cdot, t) \in C^{n}(\Omega)$ for each $t$ and $f(r, z, \cdot) \in$ $C^{m}[(0, \infty)]$ for each $r, z$. The vector $\left(n_{r}, n_{z}\right)$ is the outward unit vector normal to the boundary $\partial \Omega$.

Definition 1. A pair $(\underline{u}, \underline{\varphi})$ of the form (2.1) corresponds to a solution of the second axisymmetric initial-boundary value problem of homogeneous isotropic micropolar elastodynamics if the pair satisfies the following field equations in $\Omega \times(0, \infty)$, initial conditions in $\Omega$, and boundary conditions on $\partial \Omega \times(0, \infty)($ cf. $[17$, p. 16$],[3],[5$, p. 150$])$ :

- The equations of motion:

$$
\begin{aligned}
& 2 \sigma_{[\theta z]}+\partial_{r} \mu_{r r}+\partial_{z} \mu_{z r}+r^{-1}\left(\mu_{r r}-\mu_{\theta \theta}\right)+Y_{r}=J \ddot{\varphi}_{r}, \\
& 2 \sigma_{[r \theta]}+\left(r^{-1}+\partial_{r}\right) \mu_{r z}+\partial_{z} \mu_{z z}+Y_{z}=J \ddot{\varphi}_{z}, \\
& \partial_{r} \sigma_{r \theta}+\partial_{z} \sigma_{z \theta}+2 r^{-1} \sigma_{(r \theta)}+X_{\theta}=\varrho \ddot{u}_{\theta} \quad \text { in } \Omega \times(0, \infty) .
\end{aligned}
$$

Here $\varrho$ is the density, $J$ is the microdensity of the medium, $\left(0, X_{\theta}, 0\right)$ is the body force vector and $\left(Y_{r}, 0, Y_{z}\right)$ is the body moment vector. The parentheses (...) and brackets [...] denote the symmetric and antisymmetric parts of a tensor, respectively. $\underset{\sim}{\sigma}$ and $\underset{\sim}{\mu}$ are the force and couple stress tensor fields, respectively. In matrix form these two tensor take the form

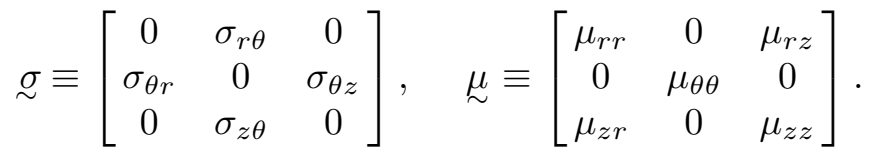


- The compatibility equations:

$$
\begin{aligned}
& \partial_{z}\left(\gamma_{r \theta}+\gamma_{\theta r}\right)+\left(r^{-1}-\partial_{r}\right)\left(\gamma_{z \theta}+\gamma_{\theta z}\right)=0, \\
& \partial_{z} \gamma_{\theta r}+r^{-1} \gamma_{z \theta}-\kappa_{z z}-\kappa_{\theta \theta}=0, \\
& \partial_{r}\left(r \gamma_{\theta r}\right)+\gamma_{r \theta}-r \kappa_{r z}=0, \\
& \kappa_{r r}+\partial_{r} \gamma_{\theta z}=0, \\
& \kappa_{\theta \theta}+r^{-1} \gamma_{\theta z}=0, \\
& \kappa_{z z}+\partial_{z} \gamma_{\theta z}=0 \quad \text { in } \Omega \times[0, \infty) .
\end{aligned}
$$

Here $\underset{\sim}{\gamma}$ and $\underset{\sim}{\kappa}$ are the strain and microstrain tensor fields respectively, and

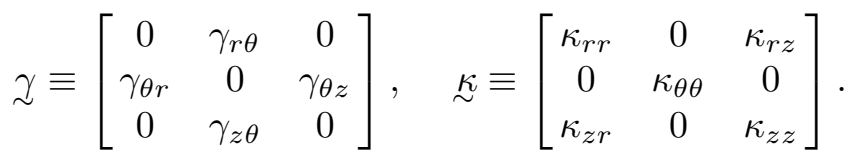

- The constitutive relations:

$$
\begin{aligned}
\mu_{r r} & =2 \gamma \kappa_{r r}+\beta \kappa, \\
\mu_{\theta \theta} & =2 \gamma \kappa_{\theta \theta}+\beta \kappa, \\
\mu_{z z} & =2 \gamma \kappa_{z z}+\beta \kappa, \\
\mu_{r z} & =(\gamma+\varepsilon) \kappa_{r z}+(\gamma-\varepsilon) \kappa_{z r}, \\
\mu_{z r} & =(\gamma+\varepsilon) \kappa_{z r}+(\gamma-\varepsilon) \kappa_{r z}, \\
\sigma_{r \theta} & =(\mu+\alpha) \gamma_{r \theta}+(\mu-\alpha) \gamma_{\theta r}, \\
\sigma_{\theta r} & =(\mu+\alpha) \gamma_{\theta r}+(\mu-\alpha) \gamma_{r \theta}, \\
\sigma_{\theta z} & =(\mu+\alpha) \gamma_{\theta z}+(\mu-\alpha) \gamma_{z \theta}, \\
\sigma_{z \theta} & =(\mu+\alpha) \gamma_{z \theta}+(\mu-\alpha) \gamma_{\theta z} \quad \text { in } \Omega \times[0, \infty),
\end{aligned}
$$

where $\kappa=\kappa_{r r}+\kappa_{\theta \theta}+\kappa_{z z}$, and $\mu, \alpha, \beta, \gamma, \varepsilon, \in \mathbb{R}_{+}$are material constants.

- The kinematic relations:

$$
\begin{aligned}
\kappa_{r r} & =\partial_{r} \varphi_{r}, \\
\kappa_{\theta \theta} & =r^{-1} \varphi_{r}, \\
\kappa_{z z} & =\partial_{z} \varphi_{z}, \\
\kappa_{r z} & =\partial_{r} \varphi_{z}, \\
\kappa_{z r} & =\partial_{z} \varphi_{r}, \\
\gamma_{r \theta} & =\partial_{r} u_{\theta}-\varphi_{z}, \\
\gamma_{\theta r} & =\varphi_{z}-r^{-1} u_{\theta}, \\
\gamma_{z \theta} & =\partial_{z} u_{\theta}+\varphi_{r}, \\
\gamma_{\theta z} & =-\varphi_{r} \quad \text { in } \Omega \times(0, \infty) .
\end{aligned}
$$


- The initial-boundary conditions:

$$
\begin{array}{llll}
\varphi_{r}=h_{r}, & u_{\theta}=h_{\theta}, & \varphi_{z}=h & \text { in } \Omega \times\{0\}, \\
\dot{\varphi}_{r}=l_{r}, & \dot{u}_{\theta}=l_{\theta}, & \dot{\varphi}_{z}=l_{z} & \text { in } \Omega \times\{0\},
\end{array}
$$

where $h_{r}, h_{z}, h_{\theta}, l_{r}, l_{z}, l_{\theta}: \Omega \rightarrow \mathbb{R}$ are given functions;

$$
\begin{aligned}
& n_{r} \mu_{r r}+n_{z} \mu_{z r}=m_{r}, \\
& n_{r} \mu_{r z}+n_{z} \mu_{z z}=m_{z}, \\
& n_{r} \sigma_{r \theta}+n_{z} \sigma_{z \theta}=p_{\theta} \quad \text { in } \partial \Omega_{\sigma} \times[0, \infty),
\end{aligned}
$$

and

$$
\varphi_{r}=\widehat{\varphi}_{r}, \quad u_{\theta}=\widehat{u}_{\theta}, \quad \varphi_{z}=\widehat{\varphi}_{z} \quad \text { on } \partial \Omega_{u} \times[0, \infty),
$$

where the functions $m_{r}, p_{\theta}, m_{z}: \partial \Omega_{\sigma} \times[0, \infty) \rightarrow \mathbb{R}$ and $\widehat{\varphi}_{r}, \widehat{u}_{\theta}, \widehat{\varphi}_{z}: \partial \Omega_{u} \times$ $[0, \infty) \rightarrow \mathbb{R}$ are given, and

$$
\partial \Omega=\partial \Omega_{\sigma} \cup \partial \Omega_{u}, \quad \partial \Omega_{\sigma} \cap \partial \Omega_{u}=\emptyset .
$$

Clearly, the problem described by $(2.2)-(2.12)$ is a mixed problem due to the mixed boundary conditions (2.10)-(2.11).

Definition 2. By a stress field corresponding to a solution of the mixed problem $(2.2)-(2.12)$ we mean a pair $\underset{\sim}{\sigma}, \underset{\sim}{\mu})$ of matrices of type (2.3) with the property that there exists an array of functions $(\underline{u}, \underline{\varphi}, \underset{\sim}{\sim}, \underset{\sim}{\sim})$ consistent with (2.1) and (2.5) such that $(\underline{u}, \underline{\varphi}, \underset{\sim}{\gamma}, \underset{\sim}{\sim}, \sigma, \underline{\sim})$ is a solution of $(2.2)-(2.12)$.

Note that one way of obtaining a triplet $\left(\varphi_{r}, u_{\theta}, \varphi_{z}\right)$ from a pair $(\mathcal{\sim}, \underset{\sim}{\sim})$ is by integrating (2.7) with respect to $r$ and $z$. As a result we get

$$
\left\{\begin{array}{l}
\varphi_{r}=-\gamma_{\theta z}, \\
u_{\theta}=r\left(\varphi_{z}-\gamma_{\theta r}\right), \\
\varphi_{z}=\int_{z_{0}}^{z} \kappa_{z z} d z+C_{1}(r, t)=\int_{r_{0}}^{r} \kappa_{r z} d r+C_{2}(z, t),
\end{array}\right.
$$

where $\left(r_{0}, z_{0}\right)$ is a fixed point of $\Omega$, and the functions $C_{1}(r, t)$ and $C_{2}(r, t)$ are determined from the compatibility equations (2.4). In Section 4 we offer an alternative method of recovering $\left(\varphi_{r}, u_{\theta}, \varphi_{z}\right)$ from $(\mathcal{\sim}, \underset{\sim}{\sim})(\mathrm{cf} .(4.7))$.

\section{Displacement-rotation field equations for the second axisym-} metric problem of micropolar elastodynamics. By eliminating $\underset{\sim}{\mathcal{N}} \underset{\sim}{\kappa}, \sigma$ and $\underset{\sim}{\mu}$ from (2.2), (2.4), (2.6) and (2.7) we arrive at the equations of motion in terms of displacements and rotations:

$$
\begin{aligned}
& \square_{4}^{0} \varphi_{r}+(\beta+\gamma-\varepsilon) \partial_{r} \kappa-2 \alpha \partial_{z} u_{\theta}+Y_{r}=0, \\
& \square_{4} \varphi_{z}+(\beta+\gamma-\varepsilon) \partial_{z} \kappa+2 \alpha r^{-1} \partial_{r}\left(r u_{\theta}\right)+Y_{z}=0, \\
& \square_{2}^{0} u_{\theta}+2 \alpha\left(\partial_{z} \varphi_{r}-\partial_{r} \varphi_{z}\right)+X_{\theta}=0 \quad \text { in } \Omega \times(0, \infty),
\end{aligned}
$$


where $\kappa=r^{-1} \partial_{r}\left(r \varphi_{r}\right)+\partial_{z} \varphi_{z}, \square_{2}^{0}=(\mu+\alpha) \nabla_{0}^{2}-\varrho \partial_{t}^{2}, \square_{4}=(\gamma+\varepsilon) \nabla^{2}-$ $4 \alpha-J \partial_{t}^{2}, \square_{4}^{0}=(\gamma+\varepsilon) \nabla_{0}^{2}-4 \alpha-J \partial_{t}^{2}, \nabla^{2}=\partial_{r}^{2}+r^{-1} \partial_{r}+\partial_{z}^{2}, \nabla_{0}^{2}=\nabla^{2}-r^{-2}$ and $\partial_{t}^{2} f=\ddot{f}$.

Definition 3. An array of functions

$$
\varphi_{r}, \varphi_{z}, u_{\theta}, \sigma, \underset{\sim}{\mu}, \underset{\sim}{\sim}, \underset{\sim}{\kappa}: \bar{\Omega} \times[0, \infty) \rightarrow \mathbb{R}
$$

that satisfies (2.1)-(2.12) is called an elastic process (or elastodynamic state; cf. [14]) of micropolar elastodynamics corresponding to the second axisymmetric problem of the theory.

Clearly, such a process may be characterized in terms of a pair $(\underline{u}, \underline{\varphi})$ only if suitable displacement-rotation initial-boundary conditions consistent with (2.8)-(2.12) are associated with the field equations (3.1).

\section{Stress equations of motion for the second axisymmetric prob- \\ lem of micropolar elastodynamics}

Definition 4. When $\partial \Omega_{u}=\partial \Omega\left(\partial \Omega_{\sigma}=\emptyset\right)$ in (2.10)-(2.12) we refer to a displacement-rotation problem. When $\partial \Omega_{\sigma}=\partial \Omega\left(\partial \Omega_{u}=\emptyset\right)$ in (2.10)(2.12) we refer to a traction problem.

By eliminating $\underline{u}$ and $\underline{\varphi}$ from (2.2) and (2.7) we obtain

$$
\begin{aligned}
& J^{-1}\left(-1, \partial_{r}, r^{-1}, \partial_{z}\right) R_{r}=\left(\ddot{\gamma}_{\theta z}, \ddot{\kappa}_{r r}, \ddot{\kappa}_{\theta \theta}, \ddot{\kappa}_{z r}\right), \\
& J^{-1}\left(\partial_{z}, \partial_{r}\right) R_{z}=\left(\ddot{\kappa}_{z z}, \ddot{\kappa}_{r z}\right), \\
& \varrho^{-1} \partial_{r} R_{\theta}-J^{-1} R_{z}=\ddot{\gamma}_{r \theta}, \\
& J^{-1} R_{z}-\varrho^{-1} r^{-1} R_{\theta}=\ddot{\gamma}_{\theta r}, \\
& \varrho^{-1} \partial_{z} R_{\theta}+J^{-1} R_{r}=\ddot{\gamma}_{z \theta} \quad \text { in } \Omega \times(0, \infty),
\end{aligned}
$$

where

$$
\begin{aligned}
& R_{r}=\widehat{R}_{r}+Y_{r}, R_{z}=\widehat{R}_{z}+Y_{z}, R_{\theta}=\widehat{R}_{\theta}+X_{\theta}, \\
& \widehat{R}_{r}=2 \sigma_{[\theta z]}+\partial_{r} \mu_{r r}+\partial_{z} \mu_{z r}+r^{-1}\left(\mu_{r r}-\mu_{\theta \theta}\right), \\
& \widehat{R}_{z}=2 \sigma_{[r \theta]}+\left(r^{-1}+\partial_{r}\right) \mu_{r z}+\partial_{z} \mu_{z z}, \\
& \widehat{R}_{\theta}=\partial_{r} \sigma_{r \theta}+\partial_{z} \sigma_{z \theta}+2 r^{-1} \sigma_{(r \theta)} .
\end{aligned}
$$

By solving (2.6) with respect to $(\mathcal{\sim}, \kappa)$ we get

$$
\begin{aligned}
& 2 \gamma \kappa_{r r}=\mu_{r r}-\beta \kappa, \\
& 2 \gamma \kappa_{\theta \theta}=\mu_{\theta \theta}-\beta \kappa, \\
& 2 \gamma \kappa_{z z}=\mu_{z z}-\beta \kappa,
\end{aligned}
$$




$$
\begin{aligned}
& \left(\kappa_{r z}, \kappa_{z r}\right)=\frac{1}{2 \gamma} \mu_{(r z)} \pm \frac{1}{2 \varepsilon} \mu_{[r z]}, \\
& \left(\gamma_{r \theta}, \gamma_{\theta r}\right)=\frac{1}{2 \mu} \sigma_{(r \theta)} \pm \frac{1}{2 \alpha} \sigma_{[r \theta]}, \\
& \left(\gamma_{\theta z}, \gamma_{z \theta}\right)=\frac{1}{2 \mu} \sigma_{(\theta z)} \pm \frac{1}{2 \alpha} \sigma_{[\theta z]} \quad \text { in } \Omega \times[0, \infty),
\end{aligned}
$$

where $\kappa=\frac{1}{2 \gamma+3 \beta}\left(\mu_{r r}+\mu_{\theta \theta}+\mu_{z z}\right)$. Next, substituting (4.2) into (4.1) and separating the equations we obtain

$$
\begin{aligned}
& 2 \gamma J^{-1} \partial_{r} R_{r}-\ddot{\mu}_{r r}+\beta \ddot{\kappa}=0, \\
& 2 \gamma J^{-1} r^{-1} R_{r}-\ddot{\mu}_{\theta \theta}+\beta \ddot{\kappa}=0, \\
& 2 \gamma J^{-1} \partial_{z} R_{z}-\ddot{\mu}_{z z}+\beta \ddot{\kappa}=0, \\
& J^{-1} \partial_{z} R_{r}-\frac{1}{2 \gamma} \ddot{\mu}_{(r z)}+\frac{1}{2 \varepsilon} \ddot{\mu}_{[r z]}=0, \\
& J^{-1} \partial_{z} R_{r}-\frac{1}{2 \gamma} \ddot{\mu}_{(r z)}+\frac{1}{2 \varepsilon} \ddot{\mu}_{[r z]}=0, \\
& J^{-1} \partial_{r} R_{z}-\frac{1}{2 \gamma} \ddot{\mu}_{(r z)}-\frac{1}{2 \varepsilon} \ddot{\mu}_{[r z]}=0, \\
& J^{-1} R_{r}+\frac{1}{2 \mu} \ddot{\sigma}_{(\theta z)}+\frac{1}{2 \alpha} \ddot{\sigma}_{[\theta z]}=0, \\
& \varrho^{-1} \partial_{r} R_{\theta}-J^{-1} R_{z}-\frac{1}{2 \mu} \ddot{\sigma}_{(r \theta)}-\frac{1}{2 \alpha} \ddot{\sigma}_{[r \theta]}=0, \\
& J^{-1} R_{z}-\varrho^{-1} r^{-1} R_{\theta}-\frac{1}{2 \mu} \ddot{\sigma}_{(r \theta)}+\frac{1}{2 \alpha} \ddot{\sigma}_{[r \theta]}=0, \\
& \varrho^{-1} \partial_{z} R_{\theta}+J^{-1} R_{r}-\frac{1}{2 \mu} \ddot{\sigma}_{(\theta z)}+\frac{1}{2 \alpha} \ddot{\sigma}_{[\theta z]}=0 \quad \text { in } \Omega \times(0, \infty),
\end{aligned}
$$

where $\ddot{\kappa}=\frac{1}{2 \gamma+3 \beta}\left(\ddot{\mu}_{r r}+\ddot{\mu}_{\theta \theta}+\ddot{\mu}_{z z}\right)$. These equations constitute the stress equations of motion for the problem under consideration. The initial conditions for a pair $(\underset{\sim}{\sigma}, \underset{\sim}{\mu})$ that satisfies $(4.3)$ are implied by the initial conditions (2.8) and (2.9), constitutive relations (2.6) and kinematic relations (2.7):

$$
\begin{array}{lll}
\dot{\sigma}^{0} \equiv\left[\begin{array}{ccc}
0 & \sigma_{r \theta}^{0} & 0 \\
\sigma_{\theta r}^{0} & 0 & \sigma_{\theta z}^{0} \\
0 & \sigma_{z \theta}^{0} & 0
\end{array}\right], & \stackrel{\sim}{\sim}^{0} \equiv\left[\begin{array}{ccc}
\mu_{r r}^{0} & 0 & \mu_{r z}^{0} \\
0 & \mu_{\theta \theta}^{0} & 0 \\
\mu_{z r}^{0} & 0 & \mu_{z z}^{0}
\end{array}\right], \\
\dot{\sigma}^{0} \equiv\left[\begin{array}{ccc}
0 & \dot{\sigma}_{r \theta}^{0} & 0 \\
\dot{\sigma}_{\theta r}^{0} & 0 & \dot{\sigma}_{\theta z}^{0} \\
0 & \dot{\sigma}_{z \theta}^{0} & 0
\end{array}\right], & \dot{\sim}^{0} \equiv\left[\begin{array}{ccc}
\dot{\mu}_{r r}^{0} & 0 & \dot{\mu}_{r z}^{0} \\
0 & \dot{\mu}_{\theta \theta}^{0} & 0 \\
\dot{\mu}_{z r}^{0} & 0 & \dot{\mu}_{z z}^{0}
\end{array}\right],
\end{array}
$$

where

$$
\left(\mu_{r r}^{0}, \mu_{\theta \theta}^{0}, \mu_{z z}^{0}\right)=2 \gamma\left(\partial_{r} h_{r}, r^{-1} h_{r}, \partial_{z} h_{z}\right)+\beta \kappa^{0}
$$


[cont.]

$$
\left(\mu_{r z}^{0}, \mu_{z r}^{0}\right)=(\gamma+\varepsilon)\left(\partial_{r} h_{z}, \partial_{z} h_{r}\right)+(\gamma-\varepsilon)\left(\partial_{z} h_{r}, \partial_{r} h_{z}\right),
$$$$
\left(\sigma_{r \theta}^{0}, \sigma_{\theta r}^{0}\right)=(\mu+\alpha)\left(\gamma_{r \theta}^{0}, \gamma_{z \theta}^{0}\right)+(\mu-\alpha)\left(\gamma_{\theta r}^{0}, \gamma_{r \theta}^{0}\right) \text {, }
$$

$$
\left(\sigma_{\theta z}^{0}, \sigma_{z \theta}^{0}\right)=(\mu+\alpha)\left(\gamma_{\theta z}^{0}, \gamma_{z \theta}^{0}\right)+(\mu-\alpha)\left(\gamma_{z \theta}^{0}, \gamma_{\theta z}^{0}\right) \text {, }
$$

$\gamma_{r \theta}^{0}=\partial_{r} h_{\theta}-h_{z}, \gamma_{\theta r}^{0}=-r^{-1} h_{\theta}+h_{z}, \gamma_{z \theta}^{0}=\partial_{z} h_{\theta}+h_{r}, \gamma_{\theta z}^{0}=-h_{r}, \kappa^{0}=$ $\left(r^{-1}+\partial_{r}\right) h_{r}+\partial_{z} h_{z}$ and

$$
\begin{aligned}
& \left(\dot{\mu}_{r r}^{0}, \dot{\mu}_{\theta \theta}^{0}, \dot{\mu}_{z z}^{0}\right)=2 \gamma\left(\partial_{r} l_{r}, r^{-1} l_{r}, \partial_{z} l_{z}\right)+\beta \dot{\kappa}^{0}, \\
& \left(\dot{\mu}_{r z}^{0}, \dot{\mu}_{z r}^{0}\right)=(\gamma+\varepsilon)\left(\partial_{r} l_{z}, \partial_{z} l_{r}\right)+(\gamma-\varepsilon)\left(\partial_{z} l_{r}, \partial_{r} l_{z}\right), \\
& \left(\dot{\sigma}_{r \theta}^{0}, \dot{\sigma}_{\theta r}^{0}\right)=(\mu+\alpha)\left(\dot{\gamma}_{r \theta}^{0}, \dot{\gamma}_{\theta r}^{0}\right)+(\mu-\alpha)\left(\dot{\gamma}_{\theta r}^{0}, \dot{\gamma}_{r \theta}^{0}\right), \\
& \left(\dot{\sigma}_{\theta z}^{0}, \dot{\sigma}_{z \theta}^{0}\right)=(\mu+\alpha)\left(\dot{\gamma}_{\theta z}^{0}, \dot{\gamma}_{z \theta}^{0}\right)+(\mu-\alpha)\left(\dot{\gamma}_{z \theta}^{0}, \dot{\gamma}_{\theta z}^{0}\right),
\end{aligned}
$$

$\dot{\gamma}_{r \theta}^{0}=\partial_{r} l_{\theta}-l_{z}, \dot{\gamma}_{\theta r}^{0}=-r^{-1} l_{\theta}+l_{z}, \dot{\gamma}_{z \theta}^{0}=\partial_{z} l_{\theta}+l_{z}, \dot{\gamma}_{\theta z}^{0}=-l_{r}, \dot{\kappa}^{0}=$ $\left(r^{-1}+\partial_{r}\right) l_{r}+\partial_{z} l_{z}$.

Now we present the following lemma (see [12, p. 93], [10]).

Lemma 1. Let $\underline{u}, \underline{\varphi}, \underset{\sim}{\sigma}$ and $\underset{\sim}{\mu}$ (see (2.1) and (2.3)) be smooth functions in $\bar{\Omega} \times[0, \infty)$. Then $\underline{u}, \underline{\varphi}, \underset{\sim}{\sigma}$ and $\underset{\sim}{\mu}$ satisfy the equations of motion $(2.2)$ as well as the initial conditions (2.8) and (2.9) if and only if

$$
\begin{aligned}
& \varphi_{r}=l_{r} t+h_{r}+J^{-1}\left(t * R_{r}\right), \\
& \varphi_{z}=l_{z} t+h_{z}+J^{-1}\left(t * R_{z}\right), \\
& u_{\theta}=l_{\theta} t+h_{\theta}+\varrho^{-1}\left(t * R_{\theta}\right) \quad \text { in } \bar{\Omega} \times[0, \infty),
\end{aligned}
$$

where $*$ stands for the convolution product on the t-axis (Mikusiński [15]):

$$
t * f(r, z, t)=\int_{0}^{t}(t-\tau) f(r, z, \tau) d \tau .
$$

Proof. We integrate the equations of motion (2.2) twice with respect to time and take into account the initial conditions (2.8) and (2.9) to obtain (4.7) in $\Omega \times[0, \infty)$. To show that (4.7) implies (2.2), (2.8) and (2.9) we proceed in a way similar to that of [12].

Definition 5 (Stress Equations of Motion Problem-SEMP). By the stress equations of motion problem associated with the second problem of axisymmetric micropolar elastodynamics we mean the initial-boundary value problem in which the field equations (4.3) are satisfied together with the initial conditions

$$
\sigma=\sigma^{0}, \quad \stackrel{\sim}{\sim}=\mu_{\sim}^{0}, \quad \dot{\sigma}=\dot{\sigma}^{0}, \quad \dot{\sim}=\dot{\mu}^{0} \quad \text { in } \Omega \times\{0\}
$$


the boundary conditions $(2.10)$ on $\partial \Omega_{\sigma} \times[0, \infty)$, and the boundary conditions

$$
\begin{aligned}
& l_{r} t+h_{r}+J^{-1}\left(t * R_{r}\right)=\widehat{\varphi}_{r}, \\
& l_{\theta} t+h_{\theta}+\varrho^{-1}\left(t * R_{\theta}\right)=\widehat{u}_{\theta}, \\
& l_{z} t+h_{z}+J^{-1}\left(t * R_{z}\right)=\widehat{\varphi}_{z} \quad \text { on } \partial \Omega_{u} \times[0, \infty) .
\end{aligned}
$$

THEOREM 1 (Characterization of the traction problem in terms of stress). Let the system of functions $(\underline{u}, \underline{\varphi}, \underset{\sim}{\sigma}, \underset{\sim}{\mu}, \underset{\sim}{\mathcal{\gamma}}, \underset{\sim}{\sim})$ be sufficiently smooth in $\bar{\Omega} \times$ $[0, \infty)$. More precisely, assume that:

(a) $m_{r}, p_{\theta}$ and $m_{z}$ are continuous in time and piecewise regular on $\partial \Omega \times$ $[0, \infty)$

(b) $h_{r}, h_{\theta}, h_{z}, l_{r}, l_{\theta}$ and $l_{z}$ are continuous in $\bar{\Omega}$ and of class $C^{2}$ in $\Omega$,

(c) $Y_{r}, X_{\theta}$ and $Y_{z}$ are continuous in $\bar{\Omega} \times[0, \infty)$ and of class $C^{2,0}$ in $\Omega \times[0, \infty)$,

(d) $\varphi_{r}, u_{\theta}$ and $\varphi_{z}$ are of class $C^{2}$ in $\Omega \times[0, \infty)$ and of class $C^{1}$ in $\bar{\Omega} \times[0, \infty)$,

(e) $\underset{\sim}{\sigma}$ and $\underset{\sim}{\mu}$ are of class $C^{3,2}$ in $\Omega \times[0, \infty)$,

(f) $\underset{\sim}{\sim} \underset{\sim}{\mu}, \dot{\sigma}, \underset{\sim}{\mu}, \widehat{R}_{r}, \widehat{R}_{\theta}$ and $\widehat{R}_{z}$ are continuous in $\bar{\Omega} \times[0, \infty)$.

Then the fields $\sigma$ and $\underset{\sim}{\mu}$ correspond to a solution of the traction problem if and only if the equations (4.3), the initial conditions (4.8) as well as the traction boundary conditions

$$
\begin{aligned}
& n_{r} \mu_{r r}+n_{z} \mu_{z r}=m_{r}, \\
& n_{r} \mu_{r z}+n_{z} \mu_{z z}=m_{z}, \\
& n_{r} \sigma_{r \theta}+n_{z} \sigma_{z \theta}=p_{\theta} \quad \text { on } \partial \Omega \times[0, \infty)
\end{aligned}
$$

are satisfied.

P r o of. Necessity. This follows from the fact that (2.2) and (2.6)-(2.10) imply the field equations (4.3), the traction boundary conditions (4.10) as well as the initial conditions (4.8).

Sufficiency. Assume that the stress fields $\underset{\sim}{\sigma}$ and $\underset{\sim}{\mu}$ correspond to a solution of the traction problem. Then the strain fields $(\underset{\sim}{\mathcal{N}, \underset{\sim}{\kappa})}($ see $(2.5))$ are determined from (4.2). Next, determine the displacement field $\underline{u}$ and the rotation field $\underline{\varphi}$ from (4.7), and observe that (4.7), (4.2) and (4.3) imply (2.7). Since the system of functions $\underline{u}, \underline{\varphi}, \underset{\sim}{\sigma}$ and $\underset{\sim}{\mu}$ satisfies the equations of motion (2.2) as well as the initial conditions (2.8) and (2.9) if and only

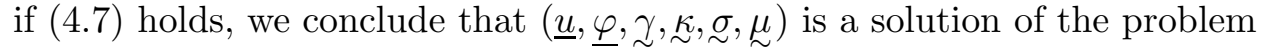
(2.2), (2.4), (2.6)-(2.9), (4.10). The proof is complete.

To complete this section, note that by integrating the stress equations of motion (4.3) twice with respect to time over the interval $[0, t]$, and taking 
into account the initial conditions, we arrive at the alternative form of stress equations of motion

$$
\begin{aligned}
& t * J^{-1} \partial_{r} R_{r}-\frac{1}{2 \gamma}\left(\mu_{r r}-\beta \kappa\right)=-\partial_{r}\left(l_{r} t+h_{r}\right), \\
& t * J^{-1} r^{-1} R_{r}-\frac{1}{2 \gamma}\left(\mu_{\theta \theta}-\beta \kappa\right)=-r^{-1}\left(l_{r} t+h_{r}\right), \\
& t * J^{-1} \partial_{z} R_{z}-\frac{1}{2 \gamma} \mu_{z z}-\beta \kappa=-\partial_{z}\left(l_{z} t+h_{z}\right), \\
& t * J^{-1} \partial_{z} R_{r}-\frac{1}{2 \gamma} \mu_{(r z)}+\frac{1}{2 \varepsilon} \mu_{[r z]}=-\partial_{z}\left(l_{r} t+h_{r}\right), \\
& t * J^{-1} \partial_{r} R_{z}-\frac{1}{2 \gamma} \mu_{(r z)}-\frac{1}{2 \varepsilon} \mu_{[r z]}=-\partial_{r}\left(l_{z} t+h_{z}\right), \\
& t * J^{-1} R_{r}+\frac{1}{2 \mu} \sigma_{(\theta z)}+\frac{1}{2 \alpha} \sigma_{[\theta z]}=-\left(l_{r} t+h_{r}\right), \\
& t * \varrho^{-1} \partial_{z} R_{\theta}+t * J^{-1} R_{r}-\frac{1}{2 \mu} \sigma_{(\theta z)}+\frac{1}{2 \alpha} \sigma_{[\theta z]} \\
& =-\partial_{z}\left(l_{\theta} t+h_{\theta}\right)-\left(l_{r} t+h_{r}\right), \\
& t * \varrho^{-1} \partial_{r} R_{\theta}-t * J^{-1} R_{z}-\frac{1}{2 \mu} \sigma_{(r \theta)}-\frac{1}{2 \alpha} \sigma_{[r \theta]} \\
& =-\partial_{r}\left(l_{\theta} t+h_{\theta}\right)+\left(l_{z} t+h_{z}\right), \\
& t * J^{-1} R_{z}-t * \varrho^{-1} r^{-1} R_{\theta}-\frac{1}{2 \mu} \sigma_{(r \theta)}+\frac{1}{2 \alpha} \sigma_{[r \theta]} \\
& =r^{-1}\left(l_{\theta} t+h_{\theta}\right)-\left(l_{z} t+h_{z}\right) \quad \text { in } \Omega \times[0, \infty) .
\end{aligned}
$$

The system (4.11) incorporates the initial conditions (4.8) explicitly in the field equations; it is a system of integro-differential equations.

THEOREM 2. Let $\underset{\sim}{\sigma}$ and $\mu$ be sufficiently smooth fields in $\bar{\Omega} \times[0, \infty)$. Then $(\sigma, \mu)$ is a stress field corresponding to a solution of the traction problem if and only if the integro-differential equations (4.11) as well as the traction boundary conditions (4.10) hold true.

Proof. By taking into account Theorem 1 it is sufficient to prove equivalence of the formulation described by (4.3), (4.8) and (4.10) with the formulation described by (4.11) and (4.10). First suppose $(\underset{\sim}{\sim}, \mu)$ meets (4.11). Define $(\mathcal{\gamma}, \approx)$ through (4.2) and $(\underline{u}, \underline{\varphi})$ through (4.7). By Theorem 1,

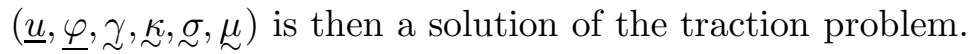


Necessity. The relations (4.3), (4.8) and (4.10), after integrating (4.3) twice with respect to time and taking into account the initial conditions (4.8), imply the field equations (4.11) and the traction boundary conditions (4.10).

Sufficiency. The equations (4.11) differentiated twice with respect to time imply the initial conditions (4.8) as well as the field equations (4.3), and the proof is complete.

Theorem 1 can be extended to a mixed problem (Gurtin [7, p. 220]).

THEOREM 3 (Characterization of the mixed problem in terms of stress). Let the system of functions $(\underline{u}, \underline{\varphi}, \underset{\sim}{\sigma}, \underset{\sim}{\mu}, \underset{\sim}{\sim}, \underset{\sim}{\sim})$ be sufficiently smooth in $\bar{\Omega} \times$ $[0, \infty)$. Then the stress fields $\sigma$ and $\underset{\sim}{\sim}$ correspond to a solution of the mixed problem if and only if the equations (4.3), the initial conditions (4.8) as well as the mixed boundary conditions (2.10) and (4.9) hold.

Proof. See [4].

5. A fundamental solution in an unbounded micropolar elastic space. Consider harmonic vibrations of an infinite micropolar space produced by the concentrated body moment

$$
Y_{z}=\frac{M_{0}}{2 \pi r} e^{-i \omega t} \delta(r) \delta(z), \quad Y_{r}=0, \quad X_{\theta}=0,
$$

where $\delta(\ldots)$ is the Dirac delta distribution, $\omega$ is the frequency of vibration and $M_{0} \in \mathbb{R}_{+}$. A fundamental solution to this problem has been obtained before by a number of authors (cf. Nowacki [17, p. 98]). In this section we obtain the solution by solving a SEMP associated with the load (5.1). Using a procedure similar to that of micropolar elastostatics (cf. [2]), from (4.3) the following uncoupled equations are obtained: the force stress equations

$$
\begin{aligned}
& \square_{3}^{0}\left(\square_{2}^{0} \square_{4}^{0}+4 \alpha^{2} \nabla_{0}^{2}\right) \sigma_{\theta z}=-2 \alpha\left[(\mu-\alpha) \square_{3}^{0}+L_{1}^{0}\right] \partial_{r z}^{2} Y_{z}, \\
& \square_{3}^{0}\left(\square_{2}^{0} \square_{4}^{0}+4 \alpha^{2} \nabla_{0}^{2}\right) \sigma_{z \theta}=-2 \alpha\left[(\mu+\alpha) \square_{3}^{0}-L_{1}^{0}\right] \partial_{r z}^{2} Y_{z},
\end{aligned}
$$

where $\square_{3}^{0}=(\beta+2 \gamma) \nabla_{0}^{2}-4 \alpha-J \partial_{t}^{2}, \square_{2}=(\mu+\alpha) \nabla^{2}-\varrho \partial_{t}^{2}$ and $\left(L_{1}, L_{1}^{0}\right)=$ $(\beta+\gamma-\varepsilon)\left(\square_{2}, \square_{2}^{0}\right)-4 \alpha^{2}$, and the couple stress equations

$$
\begin{aligned}
& \square_{3}\left(\square_{2} \square_{4}+4 \alpha^{2} \nabla^{2}\right) \mu_{z z} \\
& \quad=\left[2 \gamma L_{1} \partial_{z}^{2}-2 \gamma \square_{2} \square_{3}-\beta\left(\square_{2} \square_{4}+4 \alpha^{2} \nabla^{2}\right)\right] \partial_{z} Y_{z}, \\
& \square_{3}^{0}\left(\square_{2}^{0} \square_{4}^{0}+4 \alpha^{2} \nabla_{0}^{2}\right)\left(\mu_{r z}, \mu_{z r}\right)=\left[2 \gamma L_{1}^{0} \partial_{z}^{2}-(\gamma \pm \varepsilon) \square_{2}^{0} \square_{3}^{0}\right] \partial_{r} Y_{z},
\end{aligned}
$$

where $\square_{3}=(\beta+2 \gamma) \nabla^{2}-4 \alpha-J \partial_{t}^{2}$. In addition, the following equations are obtained:

$$
\begin{aligned}
\square_{3}\left(\square_{2} \square_{4}+4 \alpha^{2} \nabla^{2}\right) & \left(\sigma_{r \theta}-\sigma_{\theta r}\right) \\
& =-4 \alpha\left[\alpha \square_{3}\left(r^{-1} \partial_{r}+\partial_{r}^{2}\right)+L_{1} \partial_{z}^{2}-\square_{2} \square_{3}\right] Y_{z},
\end{aligned}
$$




$$
\begin{aligned}
\square_{3}\left(\square_{2} \square_{4}+4 \alpha^{2} \nabla^{2}\right)\left(\mu_{r r}+\mu_{\theta \theta}\right) \\
\quad=\left[2 \gamma L_{1}\left(r^{-1} \partial_{r}+\partial_{r}^{2}\right)-2 \beta\left(\square_{2} \square_{4}+4 \alpha^{2} \nabla^{2}\right)\right] \partial_{z} Y_{z} .
\end{aligned}
$$

Applying an integration method based on the double Fourier integral transform (with respect to the position variable $z$ and time $t$ ) and the Hankel integral transform (with respect to the position variable $r$ ) (Sneddon [19, pp. 27 and 298]) to equations (5.1)-(5.5), and using [8] we obtain the closedform results.

The force stress components $\sigma_{\theta z}$ and $\sigma_{z \theta}$ are given by

$$
\begin{aligned}
\sigma_{\theta z} & =-\frac{M_{0} e^{-i \omega t}}{4 \pi} \partial_{r z}^{2}\left(\frac{\mu-\alpha}{\mu+\alpha} \frac{p}{\lambda_{1}^{2}-\lambda_{2}^{2}} F_{1}+\frac{2 \alpha}{J \omega^{2}} G\right), \\
\sigma_{z \theta} & =-\frac{M_{0} e^{-i \omega t}}{4 \pi} \partial_{r z}^{2}\left(\frac{p}{\lambda_{1}^{2}-\lambda_{2}^{2}} F_{1}-\frac{2 \alpha}{J \omega^{2}} G\right)
\end{aligned}
$$

where

$$
\begin{aligned}
R & =\left(r^{2}+z^{2}\right)^{1 / 2}, \quad F_{1}=\frac{1}{R}\left(e^{i \lambda_{1} R}-e^{i \lambda_{2} R}\right), \\
G & =\frac{1}{R}\left[\sigma_{4}^{2}\left(\frac{A_{2}}{\lambda_{1}^{2}} e^{i \lambda_{1} R}+\frac{A_{1}}{\lambda_{2}^{2}} e^{i \lambda_{2} R}\right)-\frac{\sigma_{3}^{2}}{\lambda_{3}^{2}} e^{i \lambda_{3} R}\right], \\
\lambda_{1,2}^{2} & =\frac{1}{2}\left[\sigma_{2}^{2}+\sigma_{4}^{2}+\eta_{0}^{2}-\nu_{0}^{2} \pm \sqrt{\left(\sigma_{2}^{2}-\sigma_{4}^{2}-\eta_{0}^{2}+\nu_{0}^{2}\right)+4 \sigma_{2}^{2} \eta_{0}^{2}}\right], \\
\lambda_{3}^{2} & =\sigma_{3}^{2}-\tau_{0}^{2}, \sigma_{2}=\frac{\omega}{c_{2}}, \quad \sigma_{3}=\frac{\omega}{c_{3}}, \quad \sigma_{4}=\frac{\omega}{c_{4}}, \\
c_{2}^{2} & =\frac{\mu+\alpha}{\varrho}, \quad c_{3}^{2}=\frac{\beta+2 \gamma}{J}, \quad c_{4}^{2}=\frac{\gamma+\varepsilon}{J}, \\
\tau_{0}^{2} & =\frac{4 \alpha}{\beta+2 \gamma}, \quad \eta_{0}^{2}=p s, \quad s=\frac{2 \alpha}{\mu+\alpha}, \\
\nu_{0}^{2} & =2 p=\frac{4 \alpha}{\gamma+\varepsilon}, \quad i=\sqrt{-1}, \quad A_{1}=\frac{\sigma_{2}^{2}-\lambda_{2}^{2}}{\lambda_{1}^{2}-\lambda_{2}^{2}}, \quad A_{2}=\frac{\sigma_{2}^{2}-\lambda_{1}^{2}}{\lambda_{2}^{2}-\lambda_{1}^{2}} .
\end{aligned}
$$

The couple stress components $\mu_{z z}, \mu_{r z}$ and $\mu_{z r}$ are given by

$$
\begin{gathered}
\mu_{z z}=\frac{M_{0} e^{-i \omega t}}{4 \pi J \omega^{2}} \partial_{z}\left(2 \gamma \partial_{z}^{2} G+2 \gamma \sigma_{4}^{2} F_{2}+\beta \sigma_{3}^{2} \frac{e^{i \lambda_{3} R}}{R}\right), \\
\left(\mu_{r z}, \mu_{z r}\right)=\frac{M_{0} e^{-i \omega t}}{4 \pi J \omega^{2}} \partial_{r}\left[2 \gamma \partial_{z}^{2} G+(\gamma \pm \varepsilon) \sigma_{4}^{2} F_{2}\right]
\end{gathered}
$$

where $F_{2}=\frac{1}{R}\left(A_{1} e^{i \lambda_{2} R}+A_{2} e^{i \lambda_{1} R}\right)$.

To obtain the components $\sigma_{r \theta}, \sigma_{\theta r}, \mu_{r r}$ and $\mu_{\theta \theta}$ we integrate (5.4), (5.5) and the following equations (cf. (4.3)): 


$$
\begin{aligned}
\partial_{r}\left(r^{2} \mu_{r r}\right)= & r\left(\mu_{r r}+\mu_{\theta \theta}\right)-r^{2} \partial_{z} \mu_{z r}-2 r^{2} \sigma_{[\theta z]} \\
& -J r^{2}\left(\frac{1}{2 \mu} \ddot{\sigma}_{(z \theta)}-\frac{1}{2 \alpha} \ddot{\sigma}_{[z \theta]}\right), \\
\partial_{r z}^{2}\left(r^{2} \sigma_{r \theta}\right)= & 2 r \partial_{z} \sigma_{[r \theta]}-r^{2} \partial_{z}^{2} \sigma_{z \theta}+\frac{\varrho}{\mu} r^{2} \ddot{\sigma}_{(z \theta)} .
\end{aligned}
$$

By (5.1), (5.6) and (5.7) we obtain the force stress components

$$
\begin{aligned}
\sigma_{r \theta} & =-\frac{M_{0} \alpha e^{-i \omega t}}{2 \pi J \omega^{2}}\left[\frac{\sigma_{4}^{2}}{\lambda_{1}^{2}-\lambda_{2}^{2}}\left(\partial_{r}^{2}-\frac{\mu-\alpha}{\mu+\alpha} r^{-1} \partial_{r}\right) F_{1}+\sigma_{4}^{2} F_{2}+\partial_{z}^{2} G\right], \\
\sigma_{\theta r} & =\frac{M_{0} \alpha e^{-i \omega t}}{2 \pi J \omega^{2}}\left[\frac{\sigma_{4}^{2}}{\lambda_{1}^{2}-\lambda_{2}^{2}}\left(r^{-1} \partial_{r}-\frac{\mu-\alpha}{\mu+\alpha} \partial_{r}^{2}\right) F_{1}+\sigma_{4}^{2} F_{2}+\partial_{z}^{2} G\right]
\end{aligned}
$$

and the couple stress components

$$
\begin{aligned}
& \mu_{r r}=\frac{M_{0} e^{-i \omega t}}{4 \pi J \omega^{2}} \partial_{z}\left(2 \gamma \partial_{r}^{2} G+\beta \sigma_{3}^{2} \frac{e^{i \lambda_{3} R}}{R}\right), \\
& \mu_{\theta \theta}=\frac{M_{0} e^{-i \omega t}}{4 \pi J \omega^{2}} \partial_{z}\left(2 \gamma r^{-1} \partial_{r} G+\beta \sigma_{3}^{2} \frac{e^{i \lambda_{3} R}}{R}\right) .
\end{aligned}
$$

Formulae (5.6), (5.9) and (5.7), (5.10) determine the stress fields $\sigma$ and $\mu$, respectively, corresponding to the fundamental solution.

To obtain the displacement and rotation fields associated with the fundamental solution we use the formulae (4.7). Clearly, we need to know the initial values for the displacement and rotation fields, as well as their velocities. Combining (4.5) and (4.6) with (5.6), (5.7), (5.9) and (5.10) yields

$$
l_{r}=-i \omega h_{r}, \quad l_{z}=-i \omega h_{z}, \quad l_{\theta}=-i \omega h_{\theta}
$$

and

$$
\begin{aligned}
& h_{r}=A_{0} \partial_{r z}^{2} G, \quad h_{z}=A_{0}\left(\partial_{z}^{2} G+\sigma_{4}^{2} F_{2}\right), \\
& h_{\theta}=-\frac{2 \alpha}{\mu+\alpha} \frac{\sigma_{4}^{2}}{\lambda_{1}^{2}-\lambda_{2}^{2}} A_{0} \partial_{r} F_{1},
\end{aligned}
$$

where $A_{0}=M_{0} /\left(4 \pi J \omega^{2}\right)$.

Now using (4.7), (5.6), (5.7), (5.9)-(5.12) and the relations

$$
\begin{aligned}
& \int_{0}^{t}(t-\tau) e^{-i \omega \tau} d \tau=\omega^{-2}\left(1-i \omega t-e^{-i \omega t}\right), \\
& A_{1}+A_{2}=1 \\
& \nabla^{2}\left(\frac{e^{i \lambda_{1} R}}{R}\right)=\nabla^{2}\left(\frac{1}{R}\right)-\lambda_{1}^{2} \frac{e^{i \lambda_{1} R}}{R}
\end{aligned}
$$


we obtain the formulas for the triplet $\left(\varphi_{r}, u_{\theta}, \varphi_{z}\right)$ :

$$
\begin{aligned}
& \varphi_{r}=A(t) \partial_{r z}^{2} G, \\
& u_{\theta}=-\frac{2 \alpha}{\mu+\alpha} \frac{\sigma_{4}^{2}}{\lambda_{1}^{2}-\lambda_{2}^{2}} A(t) \partial_{r} F_{1}, \\
& \varphi_{z}=A(t)\left(\partial_{z}^{2} G+\sigma_{4}^{2} F_{2}\right),
\end{aligned}
$$

where $A(t)=A_{0} e^{-i \omega t}$.

It can easily be verified that the stress fields (5.6), (5.7), (5.9) and (5.10) as well as the displacement-rotation fields (5.14) tend to zero as $R \rightarrow \infty$. Furthermore, upon taking into account (5.1), (5.6), (5.7), (5.9), (5.10) and (5.14) and the relation

$$
\frac{1}{4 \pi} \nabla^{2}\left(\frac{1}{R}\right)=-\frac{1}{2 \pi} \delta(r) \delta(z)
$$

we verify that the equations of motion (2.2) are identically satisfied.

6. Final remarks. (i) In a SEMP described by (4.11) and (4.10) one does not have to bother with the compatibility equations (cf. (2.4)) as these are implied by (4.11).

(ii) In a SEMP the number $N$ of field equations (cf. (4.11)) is the same as the number of components of the strain state or the number of independent components of the stress state, or the number of equations of motion and compatibility equations taken together. In the problem considered, $N=9$. This result is pertinent to plane, axisymmetric and three-dimensional problems (one tensor equation corresponds to number 9) (see [5, p. 87]).

(iii) A SEMP for arbitrary initial stress fields and stress velocities also makes sense, although such a problem generally is not associated with linear micropolar elastodynamics (see [4]). In this case the compatibility conditions are not satisfied at $t=0$.

(iv) In coupled micropolar thermoelasticity an initial-boundary value problem in terms of a triplet $(\sigma, \mu, T)$ can be formulated ( $T$ is the temper-

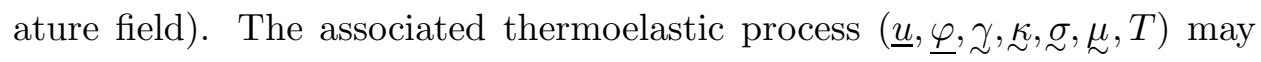
then be described in a natural way by means of the triplet $(\sigma, \mu, T)$, similarly to the isothermal theory. A SEMP with the temperature field $T$ corresponding to the displacement vector $\underline{u} \equiv\left(u_{r}, 0, u_{z}\right)$ and rotation vector $\underline{\varphi} \equiv\left(0, \varphi_{\theta}, 0\right)$ will be discussed in another paper (see [4]).

(v) The formulas (2.13) and (4.7) lead to the same triplet $\left(\varphi_{r}, u_{\theta}, \varphi_{z}\right)$ (see $(5.14))$. To prove this we note that $(2.13)_{1}$ and (4.2) yield

$$
\varphi_{r}=-\frac{1}{2 \mu} \sigma_{(z \theta)}+\frac{1}{2 \alpha} \sigma_{[\theta z]} \text {. }
$$


Substituting (5.6) into (A) we obtain the rotations $\varphi_{r}$. Next, using (2.7) and (4.2) we reduce $(2.13)_{3}$ to the form

$$
\partial_{r} \varphi_{z}=\frac{1}{2 \gamma} \mu_{(z r)}+\frac{1}{2 \varepsilon} \mu_{[r z]}, \quad \partial_{z} \varphi_{z}=\frac{1}{2 \gamma}\left(\mu_{z z}-\mu_{\theta \theta}\right)+r^{-1} \varphi_{r}
$$

Also, combining (5.7), (5.10), (5.14) 1 and (B) we arrive at

$$
\begin{aligned}
& \partial_{r}\left[\varphi_{z}-A(t)\left(\partial_{z}^{2} G+\sigma_{4}^{2} F_{2}\right)\right]=0, \\
& \partial_{z}\left[\varphi_{z}-A(t)\left(\partial_{z}^{2} G+\sigma_{4}^{2} F_{2}\right)\right]=0 .
\end{aligned}
$$

After integrating (C) we obtain the rotations $\varphi_{z}$ (cf. (5.14) $)_{3}$ ). Finally, using (4.2), we reduce $(2.7)_{7}$ to the form

$$
u_{\theta}=r\left(\varphi_{z}-\frac{1}{2 \mu} \sigma_{(r \theta)}+\frac{1}{2 \alpha} \sigma_{[r \theta]}\right) .
$$

Hence, by (5.9), (5.14) $)_{3}$ and (D) we obtain the displacements $u_{\theta}$.

(vi) The coefficient $M_{0}$ in (5.1) is introduced to comply with SI units (see [5, p. 44] and [13]), and to have the fundamental solution in a dimensional form. For example, let $[L]$ and $[P]$ denote the dimensions of LHS and RHS of $(5.14)_{2}$, respectively. We have: $\left[M_{0}\right]=[\mathrm{N} \cdot \mathrm{m}],[t]=[\mathrm{s}],[\omega]=\left[\mathrm{s}^{-1}\right]$, $\left[e^{-i \omega t}\right]=[1],[\gamma]=[\varepsilon]=[\mathrm{N}],[\alpha]=[\mu]=\left[\mathrm{N} \cdot \mathrm{m}^{-2}\right],[\varrho]=\left[\mathrm{N} \cdot \mathrm{s}^{2} \cdot \mathrm{m}^{-4}\right]$, $[J]=\left[\mathrm{N} \cdot \mathrm{s}^{2} \cdot \mathrm{m}^{-2}\right],\left[\sigma_{2}\right]=\left[\sigma_{4}\right]=\left[\eta_{0}\right]=\left[\nu_{0}\right]=\left[\mathrm{m}^{-1}\right],\left[\lambda_{1}\right]=\left[\lambda_{2}\right]=\left[\mathrm{m}^{-1}\right]$, $[R]=[\mathrm{m}],\left[e^{-i \lambda_{1} R}\right]=[1],\left[F_{1}\right]=\left[\mathrm{m}^{-1}\right],[d Y / d X]=\left[Y \cdot X^{-1}\right],\left[\partial_{r} F_{1}\right]=\left[\mathrm{m}^{-2}\right]$, $[A(t)]=\left[\mathrm{m}^{3}\right]$. Therefore $[L]=[P]=[\mathrm{m}]$.

Acknowledgements. The author would like to thank Professor Józef Ignaczak for the inspiration and for helpful discussions.

\section{References}

[1] S. Drobot, On Cosserat continua, Zastos. Mat. 12 (1971), 323-346.

[2] J. Dyszlewicz, Stress formulation of the second axially-symmetric problem of micropolar theory of elasticity, Bull. Acad. Polon. Sci. Sér. Sci. Tech. 21 (1973), 45-56.

[3] - , The stress and displacement functions for the second axisymmetric problem of micropolar elastostatics, Arch. Mech. 27 (1975), 393-404.

[4] - The problem of stress equations of motion of Ignaczak type. The axisymmetric and plane problems of micropolar elastodynamics, manuscript, IPPT PAN, Warszawa, 1987 (in Polish).

[5] _, Boundary and Initial-Boundary Value Problems for Micropolar Elastostatic and Elastodynamic Equations, WPW, Wrocław, 1990 (in Polish).

[6] A. C. Eringen, Linear theory of micropolar elasticity, J. Math. Mech. 15 (1966), 909-930.

[7] M. E. Gurtin, The Linear Theory of Elasticity, in: Encyclopedia of Physics, vol. 6a/2, Springer, Berlin, 1972. 
[8] I. S. Gradshteı̆n and I. M. Ryzhik, Tables of Integrals, Sums, Series and Products, Nauka, Moscow, 1971 (in Russian).

[9] D. Iesan, On the plane coupled micropolar thermoelasticity, Bull. Acad. Polon. Sci. Sér. Sci. Tech. 16 (1968), 379-384.

[10] - On the linear theory of micropolar elasticity, Internat. J. Engrg. Sci. 7 (1969), $1213-1220$.

[11] J. Ignaczak, A completeness problem for stress equations of motion in the linear elasticity, Arch. Mech. 15 (1963), 225-234.

[12] - , Tensorial equations of motion for elastic materials with microstructure, in: Trends in Elasticity and Thermoelasticity, Witold Nowacki Ann. Volume, WoltersNoordhoff, Groningen, 1971, 90-111.

[13] W. Kasprzak and B. Lysik, Dimensional Analysis, WNT, Warszawa, 1988 (in Polish).

[14] V. D. Kupradze, T. G. Gegelya, M. O. Baskhelishvili and T. V. Burkhuladze, Three-Dimensional Problems of the Mathematical Theory of Elasticity and Thermoelasticity, Nauka, Moscow, 1976 (in Russian).

[15] J. Mikusiński, Operational Calculus, Pergamon, New York, 1959.

[16] W. Nowacki, Theory of Elasticity, PWN, Warszawa, 1970 (in Polish).

[17] -, Theory of Asymmetric Elasticity, PWN, Warszawa, 1981 (in Polish).

[18] Z. Olesiak, Stress differential equations of the micropolar elasticity, Bull. Acad. Pol. Sci. Sér. Sci. Tech. 18 (1970), 177-184.

[19] I. N. Sneddon, The Use of Integral Transforms, McGraw-Hill, New York, 1972.

Janusz Dyszlewicz

Institute of Mathematics

Technical University of Wrocław

Wybrzeże Wyspiańskiego 27

50-370 Wrocław, Poland 